\title{
Alignment between the New Zealand Health Star Rating System and the Chilean Warning Label System ${ }^{+}$
}

\author{
Fredrik Söderlund 1,2, Helen Eyles ${ }^{2,3, *}$ and Cliona Ni Mhurchu ${ }^{2}$ \\ 1 Department of Bioscience and Nutrition, Karolinska Institutet, 14183 Huddinge, Sweden; \\ f_soderlund@hotmail.com \\ 2 National Institute for Health Innovation, University of Auckland, Auckland 1072, New Zealand; \\ c.nimhurchu@auckland.ac.nz \\ 3 Department of Epidemiology and Biostatistics, and The National Institute for Health Innovation, \\ University of Auckland, Auckland 1072, New Zealand \\ * Correspondence: h.eyles@auckland.ac.nz \\ + Presented at the 2018 Nutrition Society of New Zealand Annual Conference, Auckland, New Zealand, \\ 28-30 November 2018.
}

Published: 12 March 2019

Background: Unhealthy diet is the leading preventable risk for poor health in New Zealand. Simple, front-of-pack nutrition labels may help consumers choose healthier foods. The prevailing New Zealand front-of-pack labelling system, the Health Star Rating (HSR), rates packaged foods from half a star (least healthy) to five stars (most healthy). However, the system has received criticism for allowing some high-sugar products to display high star ratings. An alternative could be to display warnings on products high in energy, sodium, total sugars or saturated fat, such as the Chilean Warning Label system. This study aimed to determine the degree of alignment between the New Zealand HSR system and the Chilean Warning Label system.

Methods: We used the 2018 Nutritrack database containing labelling and composition information for $\sim 15,000$ packaged foods and beverages sold in major New Zealand supermarkets. The nutrient threshold criteria for the Chilean warning labels were applied to all relevant products. Results will be cross-checked against HSR product star ratings to determine the degree of alignment between the two labelling systems. Products will be classified as 'apparent outliers' when a product receives no warning labels but has a HSR of $<2$ stars, suggesting an unhealthy nutritional profile or receives $>1$ warning label but has an HSR $\geq 3.5$, suggesting a healthy nutritional profile.

Results: Analysis of the application of the warning labels is underway. Additional analysis by food group and of the alignment of the warning labels with the HSR will take place afterwards. Results will be presented for the first time at the New Zealand Nutrition Society meeting.

Conclusions: The study is applying the Chilean Warning Label system to the $\sim 15,000$ packaged foods and beverages sold in major New Zealand supermarkets. Analysis of alignment with the HSR system will inform potential improvements to food labelling in New Zealand.

Supplementary Material: The poster is available online at www.mdpi.com/2504-3900/8/1/29/s1. 